Aim Identify if each ECHO network achieved it aims and objectives.

Methods Retrospective online survey designed for each network administered after final ECHO session.

Results 27\% (60/224) registered participants across each network responded. Overall, each network achieved its aims and objectives with participants reporting increased palliative care knowledge and increased confidence in managing end of life care scenarios. Benefits included case-based learning, professional networking, and avoidance of travel to access education.

Participants reported improved communication with patients and families, and improved decision making in relation to patient care. Time restraints due to staffing issues or workload were identified as barriers to participation.

Conclusion Project ECHO may be an ideal solution for meeting the demand for palliative care education across multiple settings. To ensure practitioners fully benefit, protected time for participation is recommended.

Funders Health and Social Care Board/Hospice UK.

\section{IMPLEMENTING AN ADAPTATION OF THE CARER SUPPORT NEEDS ASSESSMENT TOOL (CSNAT) INTERVENTION TO PROVIDE TAILORED SUPPORT FOR FAMILY CARERS OF PEOPLE WITH MOTOR NEURONE DISEASE}

${ }^{1}$ Gail Ewing, ${ }^{2}$ Sarah Croke, ${ }^{2}$ Christine Rowland, ${ }^{2}$ Alex Hall, ${ }^{2}$ Gunn Grande. ${ }^{1}$ University of Cambridge, UK; ${ }^{2}$ University of Manchester, UK

\subsection{6/spcare-2019-mariecuriepalliativecare.20}

Family carers face substantial demands when supporting people with Motor Neurone Disease (MND) (Aoun et al., 2013). Timely support from healthcare professionals is crucial to enable carers to fulfil their caregiving role and to look after their own wellbeing. The CSNAT intervention is an evidence-based approach for person-centred assessment and support. This project aimed to adapt the CSNAT intervention to the context of MND care, and test its implementation within specialist MND services.

Stage 1 involved interviews and focus groups with carers $(n=33)$ to explore carers' support needs at key stages of the patient's illness; stage 2 involved workshops with carers $(n=19)$ and practitioners $(n=22)$ to adapt the content of CSNAT intervention and its delivery within MND care. Stage 3 involved implementation of the adapted CSNAT-MND in three specialist MND services, and interviews with practitioners $(n=6)$ and carers $(n=6)$ exploring their experiences of the intervention.

Content of CSNAT-MND included an additional domain on support with relationships. Intervention implementation adapted to (1) legitimise assessment of carer support needs as part of routine care in MND by practitioners, (2) deliver of a stand-alone introduction stage of the CSNATMND intervention and (3) provide a dedicated assessment and planning conversation at a subsequent contact. This basic model was then suited to fit the working practices of three different MND services. This project provides insights into how carers of people with MND want to be assessed and supported, how this process can be delivered, and the feasibility of implementing the CSNAT-MND in routine practice.

\section{THE USEFULNESS AND ACCEPTABILITY OF A PERSONAL- HEALTH RECORD TO YOUNG PEOPLE WITH A LIFE- LIMITING CONDITION: A REALIST EVALUATION}

${ }^{1}$ Janet Diffin, ${ }^{2}$ Bronagh Byrne, ${ }^{2}$ Peter O'Halloran. ${ }^{1}$ Hospice UK, UK; ${ }^{2}$ Queen's University Belfast, UK

\subsection{6/spcare-2019-mariecuriepalliativecare.21}

Background Communication between young people (YP) with life-limiting conditions and healthcare professionals (HCPs) can be challenging, particularly during transition from children to adult services. ${ }^{1}$ Personal-health records (PHRs) have the potential to increase YP's selfadvocacy ${ }^{2}$ yet have not been evaluated thoroughly with this population.

Aim Use realist evaluation to test and refine a programme theory on how PHRs are thought to work with YP with a life-limiting condition.

Methods Qualitative study informed by realist evaluation. YP aged 16-24 years old living in Northern Ireland with a lifelimiting condition were invited to use a PHR; semi-structured interviews conducted at three, six and nine months to examine acceptability and use. Framework method used for analysis.

Results Eight YP and two parents (on behalf of child) participated. 4/10 used the PHR; reasons included: (i) to help organise their care, (ii) feel more confident with communication, (iii) reduce the need to repeat details about condition(s)/ medications, and (iv) manage health care when aspects of their lives were changing. PHR use depended on the level of need, desire for autonomy/self-management, and whether it brought benefits over how they currently managed their condition. Two additional contextual considerations which influenced PHR use were identified (i) YPs previous experiences in communication with HCPs, and (ii) the timing of the introduction of the PHR.

Conclusion PHRs may be useful for YP who wish to become more independent over management of their healthcare but should be targeted towards those who identify a need, and their usage supported by HCPs.

Funder Marie Curie.

\section{WHAT DO INFORMAL CARERS OF PEOPLE LIVING WITH BREATHLESSNESS IN ADVANCED DISEASE WHAT TO LEARN ABOUT 'WHAT TO EXPECT IN THE FUTURE'?}

${ }^{1}$ Sylvia Barnes, ${ }^{2}$ Gail Ewing, ${ }^{1}$ Morag Farquhar. ${ }^{1}$ University of East Anglia, UK; ${ }^{2}$ University of Cambridge, UK

\subsection{6/spcare-2019-mariecuriepalliativecare.22}

Aim Breathlessness is distressing, disabling, and common in advanced disease. Informal carers (family/friends) can lack knowledge and confidence in caring, experiencing anxiety and uncertainty. The Learning about Breathlessness programme $(\mathrm{LaB})$ addresses this.

LaB1 identified six topics carers want to learn about breathlessness. ${ }^{1}$ LaB2 is co-developing (with carers, patients and clinicians) a prototype web-based educational intervention for carers on breathlessness. Content for five of the six topics was developed, but additional work was needed for the sixth topic, 'What to expect in the future', to ensure utility and sensitivity.

Method Two disease-specific focus groups and six interviews with bereaved carers of people with breathlessness due to 
cancer or chronic obstructive pulmonary disease $(n=12)$ identified what carers would have liked to have learnt, and sensitive ways to present this for potentially copresent patients. Transcripts analysed using content analysis; validation sought from the Carer Advisory Group (CAG) and Study Advisory Group (SAG: includes clinical experts). Follow-on workshops with current carers and patients reviewed resultant draft webpages for utility and sensitivity.

Results Regarding 'the future', carers wanted to learn about: coping with symptom deterioration, 'future' discussions with others, accessing care/support, administrative tasks (e.g. death registration), and bereavement. Findings were CAG and SAG validated. Current carers and patients provided valuable feedback on drafted webpages.

Conclusion Co-developed webpages on 'What to expect in the future' included subjects bereaved carers identified as key; current carers and patients confirmed utility and sensitivity. Follow-on work is testing the full prototype website with carers, patients and clinicians to enable refinement.

\section{REFERENCE}

1. Farquhar M, Penfold C, Benson J, Lovick R, Mahadeva R, Howson S, ... Ewing $\mathrm{G}$. Six key topics informal carers of patients with breathlessness in advanced disease want to learn about and why: MRC phase I study to inform an educational intervention. PloS One 2017;12(5):e0177081.

\section{IMPLEMENTING THE SUPPORT NEEDS APPROACH FOR PATIENTS (SNAP): PATIENT EXPERIENCES}

${ }^{1}$ Carole Gardener, ${ }^{1}$ Gail Ewing, ${ }^{2}$ Morag Farquhar. ${ }^{1}$ University of Cambridge; ${ }^{2}$ University of East Anglia

\subsection{6/spcare-2019-mariecuriepalliativecare.23}

Introduction The Support Needs Approach for Patients (SNAP) is a complex intervention designed to enable personcentred care for patients with progressive non-malignant conditions, such as chronic obstructive pulmonary disease (COPD).

SNAP consists of:

1. the validated SNAP tool: designed for purpose, comprising 15 evidence-based questions tohelp patients consider areas where they may need more support.

2. the SNAP approach: a needs-led conversation between the patient and healthcare professional (HCP) to express, prioritise and address unmet support needs identified through the patient-completed tool.

Understanding patients' experiences of the intervention is crucial.

Aim To explore patient experiences of SNAP within a pilot implementation study.

Methods SNAP-trained HCPs implemented SNAP with patients with COPD across three care settings (primary, community and secondary) in the East of England over four months $(n=56)$. Topic-guided qualitative interviews were conducted with a purposive sample of patients $(n=20)$ focusing on their experiences. Interview transcripts were analysed using thematic analysis.

Results SNAP helped patients recognise their unmet support needs and gave them 'permission' to discuss these needs with HCPs. Patients described being active participants in SNAP discussions and were positive about how identified support needs were addressed. Patients without current support needs identified SNAP's potential future utility. Patients who reported less gains were unclear about the purpose of the SNAP tool and could not recall a SNAP conversation.

Discussion Patients with COPD described a range of benefits from SNAP. Simultaneously collected HCP data is currently being analysed to enable further understanding of why some patients reported less gains.

\section{WHAT INFORMATION SHOULD HOSPICES COLLECT RELATING TO THE IMPLEMENTATION OF BEREAVEMENT SERVICES? RESULTS FROM A DELPHI SURVEY}

Mirella Longo, Stephanie Sivell, Hannah Scott, Annmarie Nelson, Anthony Byrne, Kathy Seddon, Emily Harrop. Cardiff University, UK

\subsection{6/spcare-2019-mariecuriepalliativecare.24}

Background A recent Marie Curie funded study identified two core outcomes for evaluating the impact of bereavement support on adult service users in adult palliative care. This project involved a systematic review, consensus days and a two round Delphi Survey with expert stakeholders (Harrop et al. 2018). Whilst the primary focus of the study was on establishing stakeholder agreement on service user outcomes, discussions that took place during the first consensus day also identified interest in what implementation and demographic data should be collected. The DELPHI questionnaire directed at researchers and service providers therefore also included a section around service implementation.

Methods Researcher and Service Provider respondents to the Delphi Survey were asked to rate the importance of recording information about: how the service was implemented; users' sociodemographic information; unintended negative impacts and costs and value for money. The respondents answered a five-point Likert scale. Answers were grouped into categories of low and high importance. Low importance included 'Not-important', 'Slightly important' and 'Moderately important' answers. The high importance category comprised the answers 'Important' and 'Very important'.

Results A total of 106 participants were classified as service providers. Over $80 \%$ of service providers thought it important or very important to collect information about: Accessibility of service/intervention (94\%), details around the service provided/intervention (88\%), knowledge and competency of staff/ volunteers (93\%), supportive relationships (83\%), unintended negative impact of the service (83\%) and users' satisfaction with the service (93\%). Opinion was more divided around the importance of collecting information around value for money and the environment.

In terms of user demographics respondents felt that it was very important or important to collect information about relationship to the deceased (86\%) and caring role (81\%), whereas they thought that collating information around age, gender, religion, ethnicity and socio-economic status was not paramount.

Conclusions Whilst a large percentage of respondents agree on the importance of collecting certain information, opinion is quite divided on other items, in particular socio-demographic 